\title{
Notas Sobre a Avaliação da Aprendizagem em Educação a Distância
}

\author{
Simone Becher Araujo Moraes*1
}

${ }^{1}$ Doutoranda pela Universidade Federal de Santa Maria, Av. Roraima n.1000 - Santa Maria - RS - Brasil. simonebechermor@gmail.com

\section{Resumo}

Este artigo faz uma retomada sobre a questão da avaliação em Educação a Distância, trazendo para reflexão alguns elementos teóricos e práticos dentro dessa temática, destacando suas principais características, metodologias e perspectivas. $\mathrm{O}$ presente trabalho está embasado em pesquisa bibliográfica de autores relevantes da área. A partir da coleta de dados, pôde-se verificar a preocupação com os métodos avaliativos em Educação a Distância, principalmente no que se refere à avaliação do processo de construção do conhecimento e ao acompanhamento do professor. Observou-se também certa insegurança em relação à avaliação da aprendizagem no âmbito dos ambientes virtuais de aprendizagem (AVAs), uma vez que ainda têm sido necessárias e exigidas legalmente avaliações presenciais. Este estudo pretende atentar para a importância de constantes pesquisas sobre o tema avaliação em EaD, visto que se deseja evitar a repetição dos equívocos da educação presencial, que tem sido caracterizada pelo modelo de avaliação punitiva e preocupada prioritariamente, na maioria dos casos, com resultados quantitativos.

Palavras-chave: Avaliação; Educação a Distância; Aprendizagem. 


\title{
Notes about learning evaluation in Distance Education
}

\begin{abstract}
This article is a resume of the evaluation in distance education, bringing to the debate, theoretical and practical elements. This is a review article based on conceptual national bibliographic sources. From the data collection, can be found a great concern with the evaluation methods in distance education and knowledge building. We conclude that there is some concern, regarding the assessment of learning within the framework of the AVA, as they still have been necessary and legally required classroom assessments. This study concludes to the importance of ongoing research on the subject that deal with distance education evaluation, since we wish to avoid repeating the same mistakes of the presential education, which has been characterized as a model to evaluate more concerned with the results and not with the process.
\end{abstract}

Keywords: Evaluation; Distance Education; Learning. 


\section{Introdução}

No Brasil, a Educação a Distância (EaD) tem, desde sua origem, uma função estratégica no processo de universalização e democratização do ensino, como forma de superar antigos problemas sociais, principalmente em decorrência das desigualdades regionais, que vão desde as bem conhecidas diferenças econômicas até o que diz respeito ao déficit educativo.

A evolução das tecnologias da informação e comunicação (TIC) favoreceu e impulsionou a concretização de outras formas de ensinar, sobretudo quando se trata da educação formal. Neste âmbito, a EaD aparece como uma modalidade capaz de atender às diferentes demandas por conhecimento em quase todas as áreas, bem como às necessidades formativas de estudantes em locais distantes e de forma diferenciada. Com isso, novas ferramentas são criadas e incorporadas aos processos de ensino e de aprendizagem fomentando ainda mais a necessidade de revisar tanto as antigas formas de ensinar quanto as formas e instrumentos utilizados na avaliação da aprendizagem dos alunos.

A avaliação em Educação sempre esteve, no decorrer da história, vinculada diretamente ao processo de ensino e de aprendizagem. O processo avaliativo tem sido há muito tempo alvo de críticas devido ao fato de estar, por longos períodos da história da Educação, pautado na verificação meramente quantitativa da aprendizagem.

Hoje, com o advento da EaD por meio dos ambientes virtuais de aprendizagem (AVAs), faz-se necessário um estudo mais aprofundado e reflexivo acerca dos processos avaliativos e do modo como os instrumentos de avaliação da aprendizagem são utilizados. No que se refere aos processos, passou-se a compreender a avaliação não como um momento isolado de verificação do que foi aprendido, mas sim voltada para a observação cotidiana do estudante em todos os aspectos que envolvem a aprendizagem escolar.

Sabe-se ainda que a qualidade dos instrumentos usados nesses processos pode facilitar o aprendizado, ajudando a torná-lo mais eficiente, uma vez que o aluno, dentro do contexto da EaD, é considerado mais construtor de seu aprendizado e com maiores possibilidades de autonomia, em detrimento do modelo de ensino tradicional presencial. 
Diante disso, este artigo tem como objetivo discorrer sobre alguns dos instrumentos avaliativos mais utilizados em ambientes virtuais de aprendizagem, tais como o Modular Object-Oriented Dynamic Learning Environment (Moodle), o TelEduc e o Rede CoOperativa de Aprendizagem (Rooda).

Para isso, buscaram-se estudos sobre o tema, tomando como referência as produções científicas das duas principais associações brasileiras: a Associação Brasileira de Educação a Distância (ABED) e a Associação Nacional de Pós-Graduação e Pesquisa em Educação (ANPEd). Além disso, outras fontes foram consultadas, sobretudo pesquisas na área de EaD e avaliação, buscando publicações sobre como o tema Avaliação em EaD tem sido abordado e quais os instrumentos avaliativos têm sido mais utilizados nessa modalidade de educação no contexto brasileiro.

No que diz respeito à organização e metodologia do trabalho, na primeira parte é tratada brevemente a evolução da EaD no Brasil, destacando suas bases legais e a importância que tem ganhado na atualidade. Num segundo momento, a discussão é sobre a necessidade de maior atenção com a questão da avaliação da aprendizagem nessa modalidade, suas principais características e objetivos de acordo com a concepção que a fundamenta. Na terceira parte são discutidos alguns dos instrumentos de avaliação mais utilizados em EaD e suas características, bem como alguns ambientes virtuais de aprendizagem. Ao final, abordam-se os resultados da pesquisa tendo como respaldo o referencial teórico adotado, bem como são realizadas algumas considerações que servem para dar continuidade à discussão do tema.

\section{Avaliação da aprendizagem em EaD}

Atualmente, o Brasil tem experimentado forte desenvolvimento e incentivo governamental para a implantação da modalidade EaD em diversas instituições de Ensino Superior. Além disso, temos desde 1995 a Associação Brasileira de Educação a Distância (ABED), que têm como finalidades o estudo, a pesquisa, o desenvolvimento, a promoção e a divulgação dessa modalidade de ensino e a extinta Secretaria de Educação a Distância (SEAD), que foi uma das pioneiras da modalidade a distância no nosso país.

As bases legais da EaD foram estabelecidas pela Lei de Diretrizes e Bases da Educação Nacional (LDBEN no 9.394) em dezembro de 1996; em 2005, o Decreto no 
5.622 regulamentou o Art. 80 e estabeleceu diretrizes para a educação nessa modalidade em âmbito nacional. O decreto conceitua a Educação a Distância como uma modalidade educacional na qual a mediação didático-pedagógica nos processos de ensino e aprendizagem ocorre com a utilização de meios de tecnologia de informação e de comunicação (TIC), com estudantes e professores desenvolvendo atividades educativas em lugares e/ou tempos diversos.

Tendo presentes as bases legais da EaD no Brasil, tem-se em conta que falar sobre avaliação é um assunto bastante complexo e ao mesmo tempo controverso, originando muitos vieses e remetendo a temas e debates sobre conhecimento, habilidades, conteúdos, aprendizagem, comportamentos e atitudes, entre outros tantos. Tais debates surgem a partir da necessidade de mensurar o aprendizado do aluno e analisar seu desempenho a fim de perceber suas dificuldades e dúvidas, suas formas de dialogar com os conteúdos e colegas e, por fim, atribuir-lhe uma nota, conceito ou aprovar a mudança de nível do estudante. Com isso, a avaliação vem sendo, desde os primórdios, vinculada diretamente ao processo de ensino e de aprendizagem e é um dos elementos centrais nesse processo, devendo ser contínua e fornecedora de informações que irão ajudar aluno e professor na construção do conhecimento (ARIEIRA, 2009).

A avaliação do aprendizado, sendo vista como processo contínuo, estimula o educador na busca de critérios que não só mostrarão o quanto o aluno sabe como também seu processo de construção do conhecimento, ou seja, sua trajetória da aprendizagem. De acordo com Hoffman (2001) sobre a relação entre professor e aluno quando existe um acompanhamento para ajudar no trajeto, é necessário percorrê-lo junto, sentir as dificuldades, apoiando, conversando, sugerindo rumos adequados a cada aluno. Sendo assim, a avaliação é uma tarefa didática essencial para o trabalho docente, pois apresenta uma complexidade grande de fatores, não podendo ser resumida à simples realização de provas e atribuição de notas. A mensuração fornece apenas os dados que deverão ser analisados quantitativamente (LIBÂNEO, 1991).

Além disso, a avaliação da aprendizagem em EaD deve considerar as particularidades do processo educativo nessa modalidade, assumindo características e adotando instrumentos condizentes com o que se propõe como projeto educacional.

No tocante à avaliação do desempenho do estudante de EaD, o Decreto no 5.622, em seu Art. 40, exige que sejam realizados, além do cumprimento das 
atividades programadas a distância, exames presenciais, os quais devem prevalecer sobre os demais resultados obtidos pelo estudante no processo de avaliação a distância do curso. Essa recomendação pode ser questionada por valorizar um padrão avaliativo semelhante, muitas vezes, ao adotado no ensino presencial.

Não obstante, com o crescente desenvolvimento das tecnologias da informação e da comunicação (TIC) e sua grande influência sobre o ensino, cada vez mais a maneira de ensinar e de avaliar o aprendizado por meio desses recursos deve ser pensada, refletida e debatida, não mais sob o ponto de vista da educação presencial, mas sob o ponto de vista próprio e particular da EaD, que possui metodologias e ferramentas diferenciadas, principalmente, no que diz respeito à relação aluno-professor (IMMIG, 2002).

Nessa direção, Arieira et al. (2009, p. 323) salientam que "a EaD é, portanto, uma oportunidade de aprendizado que pode facilitar a vida de muitas pessoas alijadas do conhecimento e permitir o acesso destas a um mundo de informações até então distante". Os AVA lançam mão dos recursos da internet para, de maneira integrada e virtual, possibilitar o acesso aos materiais didáticos e informações, aos arquivos, banco de dados, bem como à comunicação síncrona e assíncrona, organização e planejamento pedagógico, produção de atividades e de avaliação do aprendizado, tanto individual quanto em grupo, sendo um elemento a mais dentro do contexto da evolução da EaD no Brasil e no mundo.

Assim, é preciso que os AVA, que na EaD servem como instrumentos de ensinoaprendizagem, passem por constantes avaliações que contemplem tanto os aspectos pedagógicos quanto os aspectos tecnológicos desses ambientes. Isso quer dizer que deve haver avaliação sobre a adequação desses ambientes dentro dos objetivos da proposta educacional, bem como a existência de auxílios motivacionais para os alunos, atentando-se para o cuidado com a sobrecarga cognitiva, a fim de não prejudicar o desenvolvimento do aluno (SILVA, 2009).

Nessa direção, Kenski (2010, p. 66) afirma que "o modelo de EaD para oferta educacional de qualidade em grande escala já se apresenta como fator inovador, e fator que exige metodologias e processos de produção e desenvolvimento diferenciados". 
Diante disso, é preciso ter em conta que essa modalidade tem suas especificidades tanto no que diz respeito ao ensinar quanto ao aprender. Um dos grandes desafios impostos aos professores e tutores que nela atuam diz respeito à avaliação da aprendizagem dos estudantes. Acompanhar e avaliar são desafios constantes e que, por isso, precisam ser mais bem compreendidos e analisados.

O acompanhamento e a avaliação da aprendizagem estão intimamente ligados aos objetivos, projetos e expectativas educacionais que se almeja construir, ou seja, uma avaliação cuidadosa, refletida e bem elaborada permite ao educador um constante acompanhamento do aluno em seu percurso de aquisição do conhecimento. Assim, segundo Almeida (2010, p. 336).

Em muitos casos, o fracasso na aprendizagem dos alunos é resultante do descuido de avaliar continuamente o próprio trabalho com a colaboração do grupo e efetuar as reformulações que considere adequadas para produzir novos saberes, analisar as produções dos colegas, emitir feedback e espelhar-se nessas produções.

Sabendo que o ato de avaliar implica vários critérios que são estabelecidos pela equipe pedagógica de determinado curso ou disciplina, seja ele na modalidade presencial ou a distância, compreende-se que a avaliação deve ser um instrumento de apoio e incentivo ao aprendizado e não apenas para medir ou classificar o aluno.

Silva (2006, p. 23) questiona o processo avaliativo e chama a atenção para o papel do professor, que deve rever sua própria atuação na sala de aula online:

A avaliação da aprendizagem na sala de aula online requer rupturas com o modelo tradicional de avaliação historicamente cristalizado na sala de aula presencial. Se o professor não quiser subutilizar as potencialidades próprias do digital online, ou se não quiser repetir os mesmos equívocos da avaliação tradicional, terá de buscar novas posturas, novas estratégias de engajamento no contexto mesmo da docência e da aprendizagem e aí redimensionar suas práticas de avaliar a aprendizagem e sua própria atuação.

O professor precisa ter em conta que o processo avaliativo em Educação a Distância pode ocorrer de três maneiras distintas, mas não excludentes, conforme estudo feito por Maia et. al. (2005): pode ser presencial; a distância e com aplicação de testes on-line ou contínua, isto é, ao longo do curso, uma vez que, no decorrer de uma disciplina ou curso, podem ser realizados outros tipos de avaliação a fim de 
contemplar mais amplamente as capacidades cognitivas de cada estudante na sua individualidade e em grupo, bem como atingir os objetivos pedagógicos desejados.

Mesmo com as mais diversas ferramentas e recursos tecnológicos tais como fóruns, chats, questionários, wiki, entre outros, disponíveis em ambientes virtuais de aprendizagem, o processo de avaliação do aprendizado do aluno tem sido um desafio, pois ainda se tem a necessidade de desenvolver e colocar em prática metodologias para avaliar de formas mais adequadas a essa modalidade de ensino. Entretanto, pesquisas e estudos no campo da avaliação vêm sendo feitos, como os realizados por Arieira et al. (2009), dentre outros autores, e têm procurado problematizar não apenas a questão da EaD, mas também como avaliar o aprendizado dos alunos dentro dessa modalidade educacional, bem como ajudar nas questões acerca das metodologias de avaliação da aprendizagem, técnicas e instrumentos, critérios e estratégias de aprendizagem, feedback aos alunos, professores e tutores, além da autoavaliação, com a finalidade de proporcionar ao aluno e à sociedade como um todo um ensino de qualidade e um aprendizado efetivo.

A seguir apresentamos alguns dos instrumentos avaliativos mais utilizados em ambientes virtuais de aprendizagem e como eles têm sido utilizados em EaD.

\section{AVA e instrumentos avaliativos}

Os ambientes virtuais de aprendizagem (AVA), de acordo com Santos (2003), são softwares que possibilitam a disponibilização de aulas ou cursos que são ministrados via internet, com possibilidade de integrar diversos recursos e linguagens, permitem a interação entre as pessoas e o acesso à informação, possibilitando-lhes transformar essa informação em conhecimento, bem como compartilhar diversas produções.

Na compreensão de Santos (2003, p. 2), um ambiente virtual é

um espaço fecundo de significação onde seres humanos e objetos técnicos interagem potencializando, assim, a construção do conhecimento, logo a aprendizagem. Então todo ambiente virtual é um ambiente de aprendizagem? Se entendermos aprendizagem como um processo sociotécnico em que os sujeitos interagem na e pela cultura sendo esta um campo de luta, poder, diferença e significação, espaço para construção de saberes e conhecimento, então podemos afirmar que sim. 
Uma das características dos AVAs é a possibilidade de interação que pode ocorrer por meio de várias ferramentas e uma interface gráfica, que juntas permitem valorização e otimização dessa interação. Essas ferramentas de comunicação podem ser síncronas ou assíncronas; um exemplo de ferramenta síncrona é o chat, que possibilita comunicação em tempo real, diferentemente das ferramentas assíncronas, que não ocorrem necessariamente em tempo real, como, por exemplo, os fóruns, email, blog etc. (PAIVA, 2010).

De acordo com Milligam (1999), para a gestão do aprendizado e a disponibilização de materiais, um ambiente virtual de aprendizagem deve apresentar algumas funcionalidades básicas essenciais, como:

- Gestão da disponibilidade de materiais do curso;

- Controle de acesso: geralmente baseado em senha;

- Administração: acompanhamento dos alunos e registro de sua evolução;

- Calendário para acompanhamento de datas importantes;

- Avaliação: em geral formativa;

- Comunicação: em vários níveis: uma para um, um para muitos, síncronas e assíncronas;

- Espaço pessoal para os participantes, com troca de materiais e armazenamento;

- Bases de recurso: em geral menos formais do que os materiais de aprendizagem, uma espécie de banco de dados que possa ser acessado por pesquisa de palavra;

- Instalações de apoio on-line;

- Ferramentas de manutenção para a criação e atualização do material didático.

Em relação às ferramentas utilizadas para avaliar o desempenho do aluno, que em geral encontramos na maioria dos AVA, temos os testes on-line, os trabalhos colaborativos (wiki), os glossários, enquetes e questionários, bem como ferramentas 
que permitem ao administrador visualizar o perfil do aluno, monitorar seus dados, participações, frequência e gerar relatórios de suas atividades (PAIVA, 2010). Essas ferramentas variam de ambiente para ambiente; não obstante, estão em constante aprimoramento para oferecer aos cursos inovações que venham colaborar com a dinamicidade e a eficiência da troca de informações e construção do saber.

Nunes (2010, p. 3) identificou os instrumentos de avaliação mais utilizados na EaD, conforme o Quadro 1.

Quadro 1: Formas de avaliação em EaD.

\begin{tabular}{|l|c|}
\hline Respostas & Uso \% \\
\hline Fórum de discussão & 90 \\
\hline Produção de textos diversos & 74 \\
\hline Prova com supervisão & 66 \\
\hline Lista de exercícios & 59 \\
\hline Questionários & 49 \\
\hline Elaboração de projetos & 46 \\
\hline Chat & 45 \\
\hline Elaboração de artigo & 33 \\
\hline Mapas conceituais & 15 \\
\hline Prova sem supervisão & 12 \\
\hline $\begin{array}{l}\text { Outras formas de avaliação: webquest, } \\
\text { wiki, tribunal, relatório, monografia, } \\
\text { autoavaliação, portfólio, blogs, avaliação } \\
\text { por apresentação, glossário, codificação } \\
\text { de programas de computador, estudo de } \\
\text { caso, listas de discussão, trabalhos em } \\
\text { grupo, avaliação de reação. }\end{array}$ & 30 \\
\hline
\end{tabular}

Fonte: Nunes (2010).

Os dados das respostas sinalizam que, mesmo se tratando de Educação a Distância, ainda existe resistência em lançar mão dos recursos disponíveis nos AVA para avaliar o aprendizado dos alunos; até mesmo o decreto que rege e coordena a EaD no país requer formas mais "conservadoras" de avaliação pelo Decreto no 5.622 (BRASIL, 2005):

Art.40 A avaliação do desempenho do estudante para fins de promoção, conclusão de estudos e obtenção de diplomas ou certificados dar-se-á no processo, mediante:

I - cumprimento das atividades programadas; e

II - realização de exames presenciais. 
$\S 10[\ldots]$

$\S 20$ Os resultados dos exames citados no inciso II deverão prevalecer sobre os demais resultados obtidos em quaisquer outras formas de avaliação a distância.

A partir da reflexão sobre o decreto citado, pode-se questionar o fato de trabalhar com uma modalidade de ensino denominada a distância, mas que, em decorrência de desconforto ou desconfiança, a avaliação ainda necessita ser presencial (SILVA; SILVA, 2008). É importante questionar o motivo pelo qual os AVAs têm ficado, na grande maioria dos cursos, desconsiderados ou pouco utilizados no processo avaliativo.

Por outro lado, sabe-se que, na medida em que os ambientes virtuais de aprendizagem possibilitam o monitoramento da participação do aluno e sua frequência ao ambiente, está-se falando sobre os dados quantitativos do ponto de vista da avaliação. Entretanto, esses dados são imprescindíveis quando aliados aos dados qualitativos, pois, ao conhecer os caminhos percorridos pelo aluno dentro dos AVAs, pode-se saber a maneira como ele constrói o seu aprendizado (KRATOCHWILL, 2009).

Segundo Bassani \& Behar (2009), existem em uso novos AVAs e novas ferramentas já bem conhecidas em universidades, como na Universidade Federal do Rio Grande do Sul (UFRGS), na Pontifícia Universidade Católica do Rio Grande do Sul (PUCRS) e na Universidade Federal de Santa Maria (UFSM). Essas ferramentas permitem a visualização da interação dos estudantes de um curso a distância e são de fundamental importância para a avaliação do aprendizado, bem como no acompanhamento do percurso de aprendizagem. Uma dessas ferramentas é o Rede CoOperativa de Aprendizagem (Rooda), que foi desenvolvido pela equipe interdisciplinar do Núcleo de Tecnologia Digital Aplicada a Educação (NUTED) da UFRGS e disponibiliza ferramentas para interação, comunicação síncrona e assíncrona e realiza a publicação de arquivos, sendo centrado no usuário, proporcionando um ambiente de colaboração e cooperação entre os participantes, embasado na teoria de aprendizagem de Piaget, ou seja, construtivista-interacionista. De acordo com Bassani e Behar (2006, p. 3), "a avaliação da aprendizagem no plano interindividual se constitui a partir da análise integrada do conteúdo da mensagem, do valor de troca e do tipo". 
Esse ambiente é atualmente utilizado tanto em cursos da graduação quanto da pós-graduação e tem funcionalidade bastante intuitiva, que pode ser personalizada dentro de opções disponibilizadas pelo programa. São 20 as funcionalidades do AVA, algumas abertas a todos os usuários independentemente de estarem matriculados em alguma disciplina; outras são liberadas mediante participação em disciplinas.

O Rooda possui dois módulos de acompanhamento ou avaliação da evolução do aluno; um deles é o de acesso e frequência, que mostra dados quantitativos, tais como: número de acessos que o aluno fez ao ambiente, postagem de mensagens, participação nas atividades etc. Desse modo, o professor pode acompanhar/verificar a participação dos alunos e pode, baseado nessas informações, elaborar propostas de intervenção, de forma que os dados quantitativos relativos a acessos podem dar subsídios a uma análise qualitativa.

Outro módulo de acompanhamento ou avaliação tem como foco a interação do aluno com e entre os outros participantes do AVA, de forma a facilitar a avaliação no que diz respeito ao teor e ao valor das contribuições de cada participante dentro do contexto das discussões (BASSANI; BEHAR, 2009).

Além disso, percebe-se que a análise do conteúdo dessas mensagens possibilita evidenciar (ou não) o aprofundamento teórico. Entretanto, o foco da avaliação da aprendizagem ainda está centrado numa perspectiva individual (BASSANI; BEHAR, 2006, p. 7).

Algumas das ferramentas avaliativas disponíveis dentro do Rooda são: a ferramenta de Bate-papo, que tem como objetivo a troca de ideias e informações entre os participantes do ambiente, de forma privada ou compartilhada. O Rooda também possui Diário de bordo, para registro das atividades; e a ferramenta Fórum, em que as mensagens podem ser ordenadas por autores, datas ou em forma de árvore, em que o professor cria os tópicos a serem abordados e faz a mediação.

Um AVA muito utilizado em cursos no Brasil é o TelEduc, que foi desenvolvido na Universidade Estadual de Campinas pelo Núcleo de Informática aplicada à Educação (NIED) e pelo Instituto de Computação e está em funcionamento desde 1998. Conforme Paiva (2010, p. 362), o TelEduc

oferece três ferramentas que são de uso obrigatório e ficam visíveis para todos (professores e alunos). São elas: a Estrutura do ambiente, com a descrição de cada parte do ambiente e das ferramentas; a Dinâmica do 
curso, com informações sobre a metodologia e a organização do curso; e a Agenda, que é a página de entrada do ambiente, em que o professor insere a programação das atividades. As outras ferramentas são optativas para o professor, que escolhe o que quiser para compor sua disciplina.

Em seu funcionamento completo, o ambiente do TelEduc oferece a possibilidade de quatro tipos de usuários com tarefas e acessos diferenciados: o administrador, o coordenador do curso, o formador e o aluno.

O TelEduc possui como principais ferramentas avaliativas:

Fóruns de discussão: são tópicos temáticos de acordo com o conteúdo do curso, por meio de postagem de mensagens e interação entre os alunos mediados pelo professor, que, a partir do conteúdo e profundidade dessas interações, pode realizar a avaliação do desempenho individual do aluno;

Grupos: permite a divisão dos alunos para a realização de tarefas e permite ao professor monitorar a realização das mesmas e o nível de participação de cada integrante;

Exercícios: possibilita criar, editar e gerenciar exercícios com questões que podem ser dissertativas, de múltipla escolha, de associar colunas e de verdadeiro/falso;

Intermap: permite aos formadores a visualização de todos os participantes do curso nas ferramentas de correio, fóruns e bate-papo.

Umas das preocupações do grupo que coordena o AVA TelEduc é uma avaliação que se dê no modo formativo, isto é, que seja de forma contínua, durante o processo de aprendizagem do aluno. A proposta desse tipo de avaliação é ajudar o aluno a aprender, de forma que essa avaliação possa ser informativa e formadora, tanto para o professor quanto para o aluno que está envolvido no processo. Um dos papéis do professor nesse tipo de formação é o feedback para o aluno, ou seja, uma orientação contínua. 
O Moodle (Modular Object-Oriented Dynamic Learning Environment) é outro AVA muito conhecido e amplamente utilizado por universidades brasileiras; ele proporciona acompanhamento detalhado do estudante por meio de relatórios de acesso. Foi desenvolvido por Martin Dougiamas em 1999 e é um ambiente de aprendizagem a distância livre (código aberto), gratuito, que pode ser baixado e utilizado por qualquer pessoa. Ele dispõe de uma série de ferramentas que podem ser selecionadas pelo professor de acordo com os objetivos pedagógicos desejados.

O ambiente virtual Moodle é mais do que um espaço de publicação de materiais, permeado por interações predefinidas, mas como um local onde o professor espelhe as necessidades de interação e comunicação que cada contexto educacional the apresente em diferentes momentos e situações (TORRES; SILVA, 2008, p. 4).

Tecnicamente, o Moodle funciona em sistema Unix, Linux, Windows, MAC OS X, Netware ou em qualquer outro sistema que suporte a linguagem PHP.

Estão disponíveis em sua interface inúmeras possibilidades para a realização de tarefas por parte dos alunos, com materiais dinâmicos (atividades) e materiais estáticos (páginas de texto, textos na web, pastas com arquivos), bem como vários recursos de moderação, monitoramento e avaliação que podem ser utilizados pelos professores, embora este AVA ainda possua recursos limitados de multimídia, baseando-se mais em ferramentas de texto.

Algumas das ferramentas disponíveis no ambiente que podem ser utilizadas para avaliar a aprendizagem do aluno são: chat, fórum, glossário, lição, enquete, questionário, tarefa, wiki, diário, trabalhos com revisão e comentários pelo professor. $\mathrm{A}$ seguir relacionam-se alguns dos instrumentos mais utilizados nesse AVA para avaliação do aprendizado dos estudantes e sua aplicabilidade e potencialidade avaliativa.

Chat: permite a realização de discussões por texto de modo síncrono, ou seja, em tempo real; pode ser utilizado para discussões sobre os temas trabalhados durante o curso, tendo como possibilidade avaliativa os fatores comunicação, compreensão, interesse, envolvimento e participação do aluno.

Tarefas: são realizadas de forma assíncrona, ou seja, offline. Nesta ferramenta são postadas atividades tais como textos, projetos, relatórios, vídeos ou imagens, sendo estipulados previamente a data e o horário de entrega. Esta é 
uma ferramenta avaliativa interessante, pois permite que o professor comente o desempenho do aluno e grave a sua nota; comentário e nota podem ser enviados automaticamente para o e-mail do aluno.

Questionários: é uma das ferramentas mais versáteis do Moodle, pois possibilita a elaboração de vários tipos de questionários, sejam eles de questões dissertativas, de marcar verdadeiro ou falso, de completar lacunas ou de múltipla escolha, dando um leque bastante grande de possibilidades para avaliar o aprendizado do aluno.

Fórum: possibilita observar e analisar a conexão nas discussões e envolvimento dos participantes, possibilitando ao professor identificar dificuldades e aprendizagens no decorrer do processo.

Wiki: ferramenta on-line feita para ser utilizada colaborativamente, pois visa à construção conjunta de um produto final, que é em geral um texto, e à aprendizagem significativa, conforme o que é combinado entre professores e alunos. De acordo com Rosado e Bohadana (2007, p. 11), "nas wikis a regra geral é não existir controle central, pois se espera que as sucessivas contribuições dos internautas sobre o texto constituam com o tempo um consenso, um texto profundo e refinado a respeito do tema proposto". Esta ferramenta possui um editor de texto com diversas opções de formatação e edição e a opção de desativar esse editor de texto de forma a trabalhar apenas com os códigos. Com essa ferramenta é possível avaliar a capacidade de argumentação do aluno, de trabalhar em grupo, de conectar ideias, de compreensão do tema que está sendo estudado etc.

Espera-se que o Moodle venha a evoluir e ampliar cada vez mais as suas funcionalidades, cabendo, aos responsáveis pelos cursos a seleção e a aplicação corretas dos instrumentos disponíveis, focadas em objetivos educacionais sólidos, a fim de aproveitar o que ele pode oferecer de melhor em termos de avaliação do aprendizado.

Essas e outras ferramentas que podem ser utilizadas para avaliar o aprendizado dos alunos estão em constante mudança, adaptação e melhoramentos, pois, em se 
tratando de um ambiente virtual de aprendizagem gratuito e aberto, centenas de programadores estão trabalhando para a sua evolução.

\section{Considerações finais}

Tendo em vista as várias possibilidades metodológicas de realizar a avaliação da aprendizagem dos alunos que fazem cursos na modalidade EaD utilizando AVA, é preciso ter em mente que avaliar é um processo empírico, moldado pelos recursos tecnológicos que estão disponíveis. O bom funcionamento dessa modalidade depende do bom uso das ferramentas mais pertinentes e mais adequadas ao contexto, objetivos e necessidades de cada curso.

Existe ainda grande resistência em implantar de forma mais efetiva os instrumentos de avaliação exclusivamente on-line, considerando o predomínio do uso das formas avaliativas consideradas "tradicionais" no cotidiano e na concepção de ensino da maioria dos profissionais e estudantes.

Após a pesquisa e desenvolvimento deste trabalho, verificou-se grande diversidade e boa qualidade de instrumentos utilizados para avaliação da aprendizagem, embora haja escassez de materiais que versem sobre o tema instrumentos de avaliação em EaD, trazendo-nos alguns questionamentos acerca dos motivos que levam à falta de pesquisas mais aprofundadas nessa área do conhecimento por parte das universidades e institutos de educação, que, mesmo fazendo uso dos AVAs, parecem deixar de lado o estudo e a reflexão sobre a forma de avaliar nesses ambientes de ensino e de aprendizagem.

Essas questões sobre a avaliação da aprendizagem remetem ao desenrolar da história da Educação no decorrer dos séculos, marcada por forte resistência ao que é novo e inovador, resistência essa que podemos perceber até hoje, em pleno século XXI, com a insistência em reforçar a tradição do ensino apreendida dos nossos docentes e formadores, tomando-a como verdade universal e em geral fechando para o novo. Essas e outras questões sobre ensino e aprendizagem foram motivadoras deste trabalho e nos impulsionam para novas pesquisas nesse âmbito. 


\section{Referências bibliográficas}

ALMEIDA, M. E. B. Currículo, Avaliação e Acompanhamento na Educação a Distância. In: MILL, D.; PIMENTEL, N. M. Educação à Distância: Desafios Contemporâneos. São Carlos: EdUFSCar, 2010.

ARIEIRA, J. O. et al. Avaliação do aprendizado via educação a distância: a visão dos discentes. Ensaio: avaliação e políticas públicas em educação, Rio de Janeiro, v. 17, no 63, jun., 2009. Acesso em 08 agosto de 2014, disponível em http://ref.scielo.org/5dhdzs

BASSANI, P. S.; BEHAR, P. A. Avaliação da aprendizagem em ambientes virtuais. In: BEHAR, P. (org.). Modelos pedagógicos em Educação a Distância. Porto Alegre: Artmed, 2009.

- Análise das interações em ambientes virtuais de aprendizagem: uma possibilidade para avaliação da aprendizagem em EAD. RENOTE - Revista Novas Tecnologias na Educação, v. 4, no 1, jul., 2006.

BRASIL. Lei de Diretrizes e Bases da Educação Nacional, no 9.394 de dezembro de 1996. Acesso em 08 agosto de 2014, disponível em http://www.planalto.gov.br/ccivil 03/leis/19394.htm

. Decreto no 5.622, de 19 de dezembro de 2005. Acesso em 08 agosto de 2014, disponível em http://www.planalto.gov.br/ccivil 03/ ato20042006/2005/Decreto/D5622.htm

HOFFMANN, J. Avaliar para promover. Porto Alegre: Mediação, 2001.

IMMIG, H. Avaliação da aprendizagem em ambientes de educação a distância. Monografia. Faculdade de Ciência da Computação do Centro Universitário Feevale. Ciência da Computação. Novo Hamburgo. 2002. 102 p. Acesso em 08 de agosto de 2014, disponível em: http://www.garcia.pro.br/orientacoes/HenriqueTC2.pdf

KENSKI, V. M. Avaliação e acompanhamento da aprendizagem em ambientes virtuais a distância. In: MILL, D.; PIMENTEL, N. M. Educação a distância: desafios contemporâneos. São Carlos: EdUFSCar, 2010. 
KRATOCHWILL, S. Avaliação da aprendizagem em uma perspectiva dialógica a partir do fórum on-line. In: SILVA, A. C. (org.). Aprendizagem em ambientes virtuais. Porto Alegre: Mediação, 2009.

LIBÂNEO, J. C. Didática. São Paulo: Cortez. 1991.

MAIA, M. C.; MENDONÇA, A. L.; GÓES, P. Metodologia de ensino e avaliação de aprendizagem. Anais: 120 Congresso Internacional de Educação a Distância. Florianópolis, $2005 . \quad$ Disponível em: http://www.abed.org.br/congresso2005/por/pdf/206tcc5.pdf

MILLIGAN, C. Delivering staff and professional development using virtual learning environments. In: The Role of Virtual Learning Environments in the Online Delivery of Staff Development. Institute for Computer Based Learning, HeriotWatt University, Riccarton, Edinburgh EH14-4AS. Oct. 1999. Disponível em: http://www.jisc.ac.uk/media/documents/programmes/jtap/jtap-044.pdf

NUNES, R. C. A avaliação em Educação a Distância é inovadora? Uma reflexão. TCC. Programa de Pós-graduação Lato Sensu em Educação a Distância da Universidade Católica de Brasília. Cabo Frio, 2010. Disponível em: http://www.abed.org.br/congresso2010/cd/152010155747.pdf

PAIVA, Vera Menezes de O. Ambientes virtuais de aprendizagem: implicações epistemológicas. Educ. Revista, Belo Horizonte, v. 26, no 3, dez. 2010.

ROSADO, L. A. S.; BOHADANA, E. A autoria coletiva na educação: análise da ferramenta wiki para cooperação e colaboração no ambiente virtual de aprendizagem Moodle. In: $5^{\circ}$ Encontro de Educação e Tecnologia de Informação e Comunicação. 2007. Acesso em: 08 de ago. 2014, disponível em: http://etic2008.files.wordpress.com/2008/11/unesaluizalexandre.pdf

SANTOS, Edméa Oliveira. Ambientes virtuais de aprendizagem: por autorias livres, plurais e gratuitas. Revista Faeba, v. 12, no 18, 2003. Disponível em: http://www.comunidadesvirtuais.pro.br/hipertexto/home/ava.pdf

SILVA, M. O fundamento comunicacional da avaliação da aprendizagem na sala de aula Online. In: SILVA, M.; SANTOS, E. (orgs.). Avaliação da aprendizagem em Educação Online. São Paulo: Loyola, 2006. 
SILVA, Â. C.; SILVA, M. T. Avaliação da aprendizagem em ambientes virtuais: rompendo as barreiras da legislação. Rio de Janeiro: Fundação Cesgranrio, 2008. ; _ Do diagnóstico às questões avaliativas: um caminho possível via prática de avaliação em Educação a Distância. Ensaio: avaliação e políticas públicas em Educação, Rio de Janeiro, v. 17, no 63, jun. 2009. Disponível em http://ref.scielo.org/zrjrdk

TELEDUC. Acesso em 8 ago. 2014, disponível em http://ggte.unicamp.br/ teleduc/pagina inicial/estrutura.php

TORRES, Aline A.; SILVA, Maria. O ambiente Moodle como apoio à Educação a Distância. In: $2^{\circ}$ Simpósio Hipertexto e Tecnologias na Educação: Multimodalidade e Ensino. Universidade Federal de Pernambuco, 2008. 